\title{
Ian Harris, Buddhism and Politics in Twentieth-Century
} Asia

Londres, Pinter, 1999, 300 p.

Raphaël Liogier

\section{(2) OpenEdition}

\section{Journals}

Édition électronique

URL : http://journals.openedition.org/assr/1234

DOI : $10.4000 /$ assr. 1234

ISSN : $1777-5825$

\section{Éditeur}

Éditions de l'EHESS

Édition imprimée

Date de publication : 1 avril 2003

Pagination : 59-157

ISBN : 2-222-96732-5

ISSN : 0335-5985

\section{Référence électronique}

Raphaël Liogier, «lan Harris, Buddhism and Politics in Twentieth-Century Asia », Archives de sciences sociales des religions [En ligne], 122 | avril - juin 2003, document 122.24, mis en ligne le 10 novembre 2005, consulté le 24 septembre 2020. URL : http://journals.openedition.org/assr/1234 ; DOI : https:// doi.org/10.4000/assr.1234 
Un autre exemple d'intolérance antireligieuse populaire est, au cours du $\mathrm{XX}^{\mathrm{e}}$ siècle, celle qui associe l'image du porc ou du chien aux musulmans de langue chinoise, les Hui, à la grande colère de ceux-ci ; quant aux attitudes anticléricales proprement dites, dirigées contre les tenants de la fonction religieuse dans l'islam chinois traditionnel, les ahong, elles sont internes, venant de réformateurs fondamentalistes (Élisabeth Allès). La campagne antichrétienne, et surtout antiprotestante, déclenchée par les étudiants de Shanghai en 1922, attaque la religion en tant qu'institution au nom de la science, puis, s'étendant dans les milieux intellectuels, elle prend une coloration nationaliste, ce qui renouvelle le répertoire de la tradition anticléricale chinoise (Marianne Bastid-Bruguière).

Les trois contributions suivantes se concentrent sur la figure du moine bouddhique. Le cliché du bonze dévoyé, commun dans la littérature populaire en langue vulgaire $\mathrm{du} \mathrm{XVI}^{\mathrm{e}}$ au $\mathrm{XVIII}{ }^{\mathrm{e}}$ siècle, transmet des messages variés, anticléricaux et même antireligieux dans les cas les plus extrêmes, mais aussi simplement érotiques, ou au contraire, reflétant l'excentricité inhérente au saint (Vincent Durand-Dastès). L'étude de la presse naissante dans la seconde moitié du XIX ${ }^{\mathrm{e}}$ siècle, notamment d'une collection du Shenbao entre 1872 et 1878 , permet de dessiner une anatomie d'un discours anticlérical florissant, qui n'est cependant pas antireligieux: les mauvais moines bouddhistes et taoïstes tombent, au fil de centaines d'articles et d'anecdotes, sous les accusations de vie dépravée, de violence, de sorcellerie (V.G.). La contribution la plus féconde en vues originales, étendues dans le temps et l'espace, concerne les opinions que les Chinois se sont faites à travers les siècles des moines adeptes du bouddhisme tibétain (Isabelle Charleux) : des étrangers propagateurs d'une version inférieure du bouddhisme, surtout dans le cas des tenants des écoles «rouges»; ou bien, par confusion entre Inde et Tibet, pays mythiques de l'Ouest, les représentants d'un bouddhisme original dit pur ; ou encore, des maîtres tantriques doués de pouvoirs paranormaux fantastiques et d'une sexualité monstrueuse, menace barbare envers l'ordre et la famille; ou bien des manipulateurs d'empereurs de Chine non-chinois, Yuan ou Qing, pour leur soutirer un coûteux patronage. $\mathrm{Au} \mathrm{XX} \mathrm{X}^{\mathrm{e}}$ siècle, le bouddhisme tantrique est à la mode, comme de nos jours en Occident, apprécié pour le faste de ses cérémonies, le charisme et les pouvoirs supranaturels des hauts réincarnés. Dans l'ensemble, le discours chinois sur les « lamas » n'est finalement pas plus agressif que celui condamnant tout autre clergé, et il est surtout une voie détournée pour critiquer la crédulité du peuple chinois et de ses puissants ; par contre certains intellectuels de la MongolieExtérieure ont penché, à la fin du XIX ${ }^{\mathrm{e}}$ siècle et au début du XXe siècle, vers un franc anticléricalisme contre leur propre Église lamaïque.

Une mutation de l'anticléricalisme s'observe dans la Chine postmaoïste autour des nouvelles techniques populaires de maîtrise du souffle, l'art du qigong, dont le Falungong est le plus récent avatar: elles sont soit condamnées au nom de la science, soit valorisées comme traditions purement chinoises; et curieusement les polémiques anticléricales dénonçant une supercherie dans l'art du souffle sont récupérées par certains maîtres du qigong afin de détruire leurs rivaux, ainsi Li Hongzhi, le fondateur du Falungong, à l'égard des autres maîtres, alors que lui-même est la cible d'un discours officiel antisectaire (David A. Palmer). En guise de conclusion, un rapprochement est suggéré entre variations de l'anticléricalisme européen et de l'anticléricalisme chinois, justifiant une démarche comparatiste (Jean Baubérot).

Les contributions sont denses dans leur brièveté, dotées de conclusions et de résumés en français et en anglais qui aident à en saisir le fil; elles sont chacune complétées par une bibliographie fouillée et une liste de caractères chinois.

Françoise Aubin.

122.24

HARRIS (Ian), éd.

Buddhism and Politics in Twentieth-Century Asia. Londres, Pinter, 1999, 300 p.

Nombre d'analyses sociologiques du bouddhisme, particulièrement de sa pénétration des contextes socioculturels occidentaux, n'investissent pas la dimension politique. Au point que l'on ait pu se poser la question : cette religiosité est-elle fondamentalement intimiste, introspective, extra-mondaine et apolitique? Outre le fait que l'apolitisme déclaré d'un acteur social n'est en rien la garantie sociologique de la nonpertinence d'une analyse politologique de son discours, de ses stratégies et de ses attitudes, il est important de rappeler que cet apolitisme déclaré - qui n'est d'ailleurs pas général même en Occident - est très loin de constituer une caractéristique essentielle du bouddhisme.

L'ouvrage dirigé par I.H. - spécialiste du bouddhisme et des mouvements sociaux témoigne de cette dimension politique explicite, à travers une multiplicité de contributions se rapportant à des aires géo-culturelles diversifiées sur l'ensemble de l'Asie. Dix aires sont ainsi envisagées : le Myanmar (ancienne Birmanie), le Cambodge, l'Inde, le Japon, la 
Corée, le Laos, le Sri Lanka, la Thaïlande, le Tibet, le Vietnam. La plupart des textes traitent de la situation des rapports entre l'État pris dans son ensemble et le bouddhisme, avec la contribution de Tessa Bartholomeusz sur le Sri Lanka («First Among Equals: Buddhism and Sri Lankan State »), celle de Donald Swearer sur la Thaïlande (Centre and Periphery: Buddhism and Politics in Modern Thailand), celle de Bruce Matthews sur le Myanmar («The Legacy of Tradition and Authority: Buddhism and the Nation in Myanmar »), celle de I.H. lui-même sur le Cambodge (« Buddhism in Extremis: The case of Cambodgia »), celle de Hiroko Kawanami sur le Japon («Japanese Nationalism and the Universal Dharma »).

La place de la variable bouddhiste dans un processus de transition politique sera aussi examiné dans la contribution de Martin Stuart-Fox («Laos: from Buddhist Kingdom to Marxist State »), enfin deux textes traitent de mouvements sociaux locaux en lutte contre un ordre politique général, qu'il s'agisse d'un mouvement politico-religieux pouvant aller de la remise en cause de la colonisation culturelle à des revendications séparatistes ou du moins autonomistes ainsi que le décrit Ronald Schwartz concernant le bouddhisme tibétain face à l'ordre chinois («Renewal and Resistance: Tibetan Buddhism in the Modern Era ») ou qu'il s'agisse de la mobilisation de populations locales à travers une entreprise de conversion au bouddhisme visant à constituer une assise nationale permettant de lutter contre l'ordre socio-politique indien traditionnel caractérisé par le système des castes (Timothy Fitzgerald, " Politics and Ambedkar Buddhism in Maharasthra »). L'étude du cas du Vietnam (Thiên Dô, «The Quest for Enlightenment and Cultural Identity: Buddhism in Contemporary Vietnam ») - même s'il est d'une certaine manière original puisque il s'agit du seul État $\mathrm{du}$ sud-est asiatique dans lequel le bouddhisme est essentiellement mahayaniste - concentre les éléments de problématiques qui ponctuent l'ensemble des études, que l'on peut synthétiser en quelques points:

- Les instances bouddhistes furent et sont encore à la fois collaboratrices et critiques du pouvoir, parfois dans le même temps suivant les groupes, dans la plupart des pays d'Asie, partagés entre des Églises officielles et des mouvements sociaux critiques de l'établissement.

- En tant que pouvoir établi ou de contrepouvoir, un grand nombre de mouvements ont été imprégnés depuis le XIX ${ }^{\mathrm{e}}$ siècle de la pensée marxiste, ou du moins ont modernisé leur histoire et leurs doctrines en les lisant à travers des grilles cognitives occidentales.
- Cette construction de bouddhismes modernes n'aurait pas été possible sans les incursions culturelles et (ou) politiques des puissances occidentales sur les terres asiatiques.

- C'est ainsi que le bouddhisme a participé à la structuration des luttes de libération anticoloniales, et joue encore un rôle important dans la définition d'une identité panasiatique.

- Les mouvances bouddhistes sont tiraillées entre leur insertion nationale-locale et leur capacité à construire une identité panasiatique, représentant le seul trait culturel-cultuel commun à la presque totalité de ce continent.

- Dans ces contextes variés le bouddhisme apparaît comme le trait d'union culturel à la fois le plus traditionnel et le plus moderne idéologiquement, ce qui lui confère une possible fonction soit de soutien efficace soit d'instance critique déstabilisatrice.

Cette interprétation moderne des textes et de l'histoire du bouddhisme, liée en particulier à la confrontation avec l'Occident colonisateur est très clairement évoquée dans le premier texte de l'ouvrage (I.H., « Buddhism and Politics in Asia: The Textual and Historical Roots »). Ce qui ne veut pas dire que les modalités de cette politisation - idéologique, ou même institutionnelle comme en Thaïlande où l'Église bouddhiste officielle est édifiée en véritable État rationnel centralisé de ses bases locales jusqu'à son sommet national - n'ont pas de racines asiatiques. L'histoire et les concepts traditionnels bouddhistes se prêtent en effet plutôt bien à ces jeux interprétatifs permettant par exemple de passer de la conception classique du dharma, la loi bouddhiste, à celle de «dharma universel » beaucoup plus occidentalisée. La volonté d'élimination pure et simple des visions traditionnalistes donne pourtant parfois lieu à des déclarations explicites à la limite de la provocation, à l'exemple de celle qui est parue en 1934 dans Viên Âm magazine, un important organe de presse vietnamien, soulignant quatre raisons principales et incontournables de la révolution bouddhiste intellectuelle, morale, sociale et politique : «1- Il y a un crise des valeurs confucéennes traditionnelles qui accompagnent une vieille culture à l'état de ruine. 2- Le bouddhisme peut poser une nouvelle fondation plus adaptée à l'identité nationale vietnamienne. 3- Le bouddhisme est compatible avec la science et peut ainsi détourner la science de ses mauvais usages. 4- Allégés de ses rituels compliqués, un bouddhisme réformé servira mieux les générations qui préfèrent une approche rationnelle et scientifique de la connaissance. » (p. 264) C'est nous qui traduisons. 
On pourra regretter cependant une trop grande indépendance des textes les uns par rapport aux autres qui finit par masquer au lecteur les liens entretenus entre les écoles et Églises bouddhistes au-delà de leurs insertions locale et nationale. Une organisation comme l'INED (International Network of Engaged Buddhists), même si elle a son siège en Thaïlande, essaime et agit au Japon, au Vietnam et même en Occident. Mais nous sommes sans doute trop sensible à une synergie, celle des processus pan-asiatiques d'occidentalisation des mouvances bouddhistes, que nous avons voulu nous-mêmes mettre en évidence dans certains de nos travaux. Il est clair, en revanche, que certains des articles présentés auraient quelque peu gagné à éviter des développements historiques pléthoriques qui finissent parfois par réduire les analyses des situations actuelles ou du moins du $\mathrm{XX}^{\mathrm{e}}$ siècle à quelques paragraphes en fin de contribution. La description de la situation coréenne d'opposition encore actuellement brûlante entre les mouvements bouddhistes et les groupes chrétiens au pouvoir est par exemple à peine évoquée.

L'objectif de l'ouvrage tel qu'il est avoué sur la quatrième de couverture est en tout cas atteint: faire la démonstration que «l'accent sur la méditation et l'entraînement mental n'est qu'une des notes de la gamme riche et complexe de la tradition historique et mondiale du bouddhisme » (traduit par nous). La dimension politique et sociale est une autre de ces notes qui se déploie à la fois à travers un ordre monastique tantôt divisé et tantôt unifié face aux institutions et idéologies dominantes, et à la fois à travers l'émergence et le développement de mouvements laïques qui pratiquent l'activisme social en prétendant s'inspirer de la Voie du Bouddha.

\section{Raphaël Liogier.}

HERVIEU-LÉGER (Danièle), WILLAIME (Jean-Paul).

Sociologies et religion; approches classiques. Paris, PUF, 2001, 289 p. (coll. « Sociologie d'aujourd'hui »).

Le présent ouvrage reproduit la substance sinon toujours la lettre - des enseignements donnés dans un séminaire de DEA commun à l'EPHE (Ve Section, «Sciences religieuses » où J.-P.W. est directeur d'études) et à l'EHESS (dans le cadre du Centre d'Études Interdisciplinaires des Faits Religieux, dirigé par D.H.-L.). Ce séminaire s'intitule «Approches et concepts fondamentaux en sciences sociales des religions ». Il se poursuit, au-delà de la publication de l'ouvrage ici commenté, toujours en sociologie des religions, mais avec une importance reconnue aux anglo-saxons; seuls des germanophones et des francophones ont été en effet retenus dans la première série d'exposés ici relevée.

Chacun des deux auteurs de ce livre a traité d'une partie des classiques retenus comme s'imposant à la culture des chercheurs en sciences sociales : ainsi D.H.-L. a écrit les chapitres consacrés à Durkheim, Halbwachs, Le Bras et Desroche ; de son côté, J.-P.W. s'est arrêté sur Marx, Tocqueville, Weber et Simmel.

L'Introduction, signée par D.H.-L. et J.-P.W. conjointement, présente un intérêt particulier : non seulement elle souligne les raisons bien connues justifiant l'étude des classiques dans notre domaine, mais elle insiste aussi sur la nécessité d'une lecture - et de la relecture constante au cours d'une vie de chercheur - du " corpus » des textes fondateurs de notre spécialité. En effet, ces écrits ont prouvé leur utilité au cours des ans et continuent de se montrer féconds dans l'étude des dossiers relatifs à notre contemporanéité elle-même. À juste titre, les deux AA. soulignent que la sociologie peut être de l'actualité ou d'un passé récent, mais encore du passé le plus éloigné. Cette remarque faite comme en passant (cf. p. 7 ad fin.) est précieuse, empêchant de confondre avec trop de gens pressés - et sûrement stressés - la sociologie avec l'étude du présent, tandis que l'histoire serait vouée au passé. Ni histoire ni sociologie n'apparaissent condamnées à aucune des tranches arbitrairement détachées de la diachronie. Ce sont simplement des manières méthodologiquement différentes d'aborder les problèmes variés que connaissent sociétés et populations.

Personne ne s'étonnera que nous partagions encore la conviction de nos deux AA. lorsqu'ils affirment que l'étude des classiques constitue un indispensable préservatif (mes collègues n'emploient pas ce terme même) à l'encontre « des formes plus ou moins sophistiquées de positivisme des données, oubliant les grandes interrogations épistémologiques et la profondeur historique des grands classiques de la sociologie » (p. 2). Et l'on ne saurait trop oublier l'intérêt que présentent également nos classiques en matière de méthodologie. L'Introduction le souligne avec raison: «relire les classiques sous l'angle de leur analyse des faits religieux s'est d'ailleurs révélé une excellente manière d'entrer dans leur problématique générale et d'exposer leur méthode » (p. 2).

Nous avons utilisé plus haut l'expression « corpus des textes fondateurs ». Nous avons ainsi donné l'impression que l'ouvrage de nos 